\title{
On subspace-disk transitivity of bilateral weighted shifts
}

\begin{abstract}
In this paper, we give some properties of subspace-disk transitive operators and use them to characterize subspace-disk transitive bilateral weighted shifts in terms of their weight sequences. As a consequence, we show that a bilateral weighted shift is subspace-disk transitive if and only if it satisýes the subspace-diskcyclic criterion. Then, we give a much simpler condition for some other cases.
\end{abstract}

Keyword: Subspace-diskcyclic operators; Subspace-disk transitive operators; Weighted shifts 